\title{
LA ALFABETIZACIÓN CIENTÍFICA DE PERSONAS ADULTAS: UN ENFOQUE COMUNICATIVO
}

\author{
MARTÍNEZ SALVÁ, F.A. y LATORRE, A. \\ Departamento de Psicología Evolutiva y de la Educación. Facultad de Psicología. \\ Av. Blasco Ibáñez, 21. 46010 Valencia.
}

\section{SUMMARY}

The article departs from the premise that the scientific literacy of adults implies a background allowing them to participate in the communicative mode (Habermas, 1987) to take a decision on the scientific/technological advances that affect us. Starting from this definition we find that the mere conceptual learning does not ensure the improvement of this attitude for participation. It could even produce the contrary effect. When the instructive design contemplates these attitudes, the result improves, although other effects and deficiencies can appear: degree of generalization and anxiety in front of participation. Our proposal is that these aspects are also kept in mind in adult scientific education.

\section{ALFABETIZACIÓN CIENTÍFICA}

Con la incorporación al sistema educativo de la Educación de Personas Adultas (EPA) se posibilita que éstas mejoren su formación y que así puedan afrontar mejor los retos que plantea una sociedad cada vez más compleja y cambiante. Uno de ellos, que debe atenderse necesariamente, es el derivado de los avances científicotecnológicos. Podríamos decir, sin temor a exagerar, que los desarrollos científicos se han convertido en el símbolo de esta sociedad. Como señala el informe Faure (1981), el desarrollo científico en este siglo ha venido caracterizado por: un crecimiento prodigioso de los conocimientos; una institucionalización de la investigación y la innovación; una aceleración potencial del cambio, de la capitalización del saber, del crecimiento del personal científico. E igualmente es notable el acortamiento constante del intervalo que separa un descubrimiento científico de su aplicación a gran escala. Las consecuencias de esta avalancha tecnológica han producido profundas transformaciones en prácticamente todos los ámbitos sociales, y las innovaciones tecnológicas envuelven cada vez más la vida cotidiana (tecnologías de producción, informática, robótica, nuevas tecnologías, etc.). Sin embargo, sobre estos desarrollos, se ha constatado que ocasionan, en muchas ocasiones, conse- cuencias que escapan a su control y producen efectos contrarios para la humanidad: deterioro medioambiental, incremento de la pobreza, guerras más devastadoras, desigualdad de recursos, repercusiones en los derechos humanos, narcotráfico, desempleo, consumismo, etc.

Debido a esta realidad, vemos necesario que tanto desde los centros de EPA como desde otras instituciones (entidades, asociaciones, etc.) se promueva una alfabetización científica de las personas adultas que les permita afrontar informada y razonadamente estas demandas sociales.

A la hora de explicitar qué entendemos por dicha alfabetización, repetiremos una observación capital para nosotros: que muchas de las consecuencias de las aplicaciones tecnológicas nos afectan directamente. A partir de aquí se define el rasgo más importante de nuestra concepción de alfabetización científica: que debe capacitar o incrementar la capacidad de los/as afectados/as para participar en todas aquellas decisiones cuyas consecuencias les conciernan. Este planteamiento se apoya básicamente en la teoría crítica, tanto a partir de la teoría de la acción comunicativa de Habermas (1987) como de la 
propuesta de responsabilidad solidaria de Apel (Cortina, 1988). Estos autores reconocen que las personas afectadas poseen competencia comunicativa para participar mediante el diálogo en la toma de deciones o establecimiento de normas. De esta manera los acuerdos morales serían los establecidos a partir de las argumentaciones y no por los intereses particulares. Es, por lo tanto, una ética discursiva, procedimental, con un carácter solidario (contempla intereses y necesidades de todos) y responsable, ya que obliga a afrontar las consecuencias de las decisiones. Se propone y desea, a partir del reconocimiento de la realidad social actual, el avance de esta participación comunicativa, en deterioro de una participación estratégica, para avanzar hacia una sociedad moralmente más desarrollada.

Desde el campo educativo, hemos observado cómo, en la educación de adultos/as, la expresión alfabetización científica (scientific literacy) reúne cada vez más trabajos publicados que abundan en la necesidad de la formación científica (Mora, 1991; Mason, 1989; Strachan, Brown; Schuller, 1988...). A partir de ellos y por lo argumentado anteriormente, nos sumamos al enfoque de ciencia-técnica-sociedad (CTS) para la enseñanza de las ciencias a las personas adultas. Así, diversos tópicos o núcleos conceptuales pueden formar parte del currículo en la EPA. Podemos poner como ejemplo la aportación de Scandrett (1994), que elaboró The Second Chance course con el objetivo de introducir los conocimientos científicos y desarrollar una comprensión de la ciencia en ciertas áreas de interés a fin de capacitar a las personas adultas para poder seguir la prensa y comprender cómo se genera el conocimiento científico, reconociendo el contexto social en el que se produce y aplica. Los tópicos que seleccionó fueron los siguientes: ¿qué es ciencia?, la experimentación científica, la capa de ozono, síntesis de proteínas, SIDA, genética y evolución, la comunicación científica y, por último, la demografía.

Como podemos apreciar, se trata de seleccionar temas de actualidad científica con importantes repercusiones desde el punto de vista social. Añadimos a continuación otros contenidos que también pueden ser considerados para su inclusión en los currículos de ciencias en la formación de personas adultas (FPA), tanto disciplinarmente como de modo interdisciplinar: usos y repercusiones sociales de la informática (comunicaciones, acoso a la intimidad, robótica, etc.); desarrollo armamentístico; el deterioro medioambiental y el subdesarrollo; nuevas posibilidades médicas (trasplantes, eutanasia, control de la reproducción); producción y consumo de energía; relaciones de la ciencia y la tecnológica con el consumismo; influencia social en el desarrollo científico y viceversa...

A la hora de establecer el currículo vemos que estos contenidos son básicos para la comprensión de la realidad científico-técnica, pero que, desde nuestro planteamiento, también lo es el tratamiento de los valores y actitudes que pueden derivarse de ellos. Así, proponemos incluir en los diseños instruccionales el respeto a la competencia comunicativa de las personas adultas y su fortalecimiento, incrementando sus recursos para apli- car una ética discursiva de participación responsable y solidaria. A partir de entonces se establecerán, por parte de las personas participantes, los valores, normas y actitudes que se juzguen más racionales en cada una de las problemáticas tratadas.

Con el objetivo de comprobar qué sucede cuando formamos personas adultas en contenidos científico-técnicos siguiendo nuestro enfoque comunicativo, realizamos una investigación experimental que exponemos a continuación.

\section{DISEÑO DE EXPERIMENTACIÓN}

Se procedió a elaborar un diseño instructivo para la alfabetización científica de personas adultas en un tema concreto (la ingeniería genética), desde un enfoque comunicativo, y se aplicó a dos grupos de EPA correspondientes al nivel de graduado escolar (grupos $\mathrm{E}_{1}$ y $\mathrm{E}_{2}$ ). Los resultados se compararon con los obtenidos en otros dos grupos que trabajaron el mismo tema pero con diseños diferentes, realizados por los profesores de adultos colaboradores según su metodología habitual (grupos denominados $\mathrm{C}_{1} \mathrm{y}_{2}$ ).

\section{Programa instructivo del tema ingeniería genética}

Las aportaciones de la teoría de la acción comunicativa nos llevaron a elegir el tema de estudio a partir de las preferencias de un grupo experimental (el tutorizado por nosotros, $\mathrm{E}_{2}$ ), en el que se pidió que se sugiriesen posibles temas de estudio relacionados con las interacciones ciencia-técnica-sociedad (CTS). Los estudiantes seleccionaron los más interesantes para ellos y, de entre éstos, elegimos el tema de ingeniería genética por una serie de razones:

- Engloba los diferentes tipos de contenidos: conceptuales, procedimentales y actitudinales.

- Cumple con los requisitos de trascendencia social, utilidad e interés para los participantes.

- Es un contenido de continua actualidad.

- Sus contenidos permiten un enfoque interdisciplinario (relaciones con otras áreas y temas: economía, política...), el tratamiento de la imagen de la ciencia y los científicos y, en relación con lo anterior, la historia de la ciencia.

- Existe facilidad para encontrar información y materiales de soporte para el estudio.

A partir de estas posibilidades confeccionamos la unidad didáctica siguiendo, en la medida de lo posible, el planteamiento CTS y las variables que, desde una óptica constructivista y del aprendizaje adulto (conocimientos previos, relaciones explícitas entre conceptos, etc.), podían ser relevantes para el aprendizaje y el cambio actitudinal. Otras características generales eran: 
- Elaboración de los textos expositivos a partir de las recomendaciones de Sanjosé y otros (1993).

- Inclusión de contenidos relativos a las repercusiones importantes de las aplicaciones científicas.

- Introducción de aspectos puntuales de otras disciplinas como la economía, por ejemplo.

- Tratamiento de la historia de la ciencia y los aspectos humanos del científico a través de una breve historia de errores científicos relacionados con el tema de estudio.

- Selección y comentarios de noticias de prensa de interés para el tema.

- Y, sobre todo, reflexión y explicitación de valores relacionados con las posibles conductas, participativa/ no participativa, ante los avances científicos.

Al profesor colaborador del grupo experimental (grupo denominado $\mathrm{E}_{1}$ ), que también utilizó el mismo diseño, se le proporcionó, además, una guía didáctica orientadora, ofreciéndole libertad para adaptar y desarrollar las variables de enseñanza-aprendizaje según considerase más conveniente.

\section{Instrumentos para la evaluación de las actitudes de responsabilidad solidaria}

Para la evaluación de las actitudes elaboramos un cuestionario que fue cumplimentado por todos los grupos. Para no influir en el diseño instructivo de los grupos control, este cuestionario fue presentado a los/as adultos/as por el propio investigador, sin que el profesor correspondiente pudiera conocerlo (salvo, lógicamente, el profesor del otro grupo experimental). Se realizó un pase previo (actitudes previas) y otro después de la intervención (actitudes finales), considerando el cambio actitudinal la diferencia entre ambas mediciones $(\mathrm{CA}=$ AF - AP).

Para su confección recogimos previamente las creencias y actitudes predominantes en grupos de adultos distintos a los seleccionados para la investigación y seguimos la teoría de la acción razonada de Fishbein y Ajzen (Escámez, 1986) para incorporar los elementos que estos autores juzgan relevantes para el cambio actitudinal y la conducta (creencias conductuales y normativas, actitudes, normas subjetivas e intención). También se consideró el estudio de Borreguero (1993) sobre las actitudes del ciudadano medio ante los descubrimientos y aplicaciones tecnológicas. Finalmente, adaptamos los aspectos seleccionados al contenido de nuestro tema experimental.

La actitud central del cuestionario, de acuerdo con las líneas teóricas que justifican este trabajo, es la responsabilidad solidaria ante los avances en genética. La definimos en los siguientes puntos (Cortina, 1993):

1. Informarse por el tema.
2. Proporcionar la opinión personal siempre que sea necesario.

3. Admitir que se realicen las aplicaciones genéticas con la intención de favorecer la salud de las personas.

4. Que no se utilicen para manipular personas con fines particulares (económicos, de poder...).

5. Que se tomen las decisiones de importancia conjuntamente con todas las personas implicadas (políticos, expertos, ciudadanía ...).

Los ítems pueden verse en el anexo 1.

\section{Muestra experimental a la que se aplicó el cuestiona- rio de evaluación actitudinal}

Se seleccionaron los cuatro grupos de cuatro centros de EPA de la provincia de Castellón. Se tuvo en cuenta que pertenecieran tanto al entorno rural como al urbano y que éstos se repartieran por igual entre los grupos control y experimental. De este modo se eligieron los grupos de dos localidades de ámbito rural (menores de 10.000 habitantes) y de otras dos urbanas (más de 20.000 habitantes). También se intentó equilibrar la especialidad y formación de los profesores participantes, de este modo, tanto en los grupos control como en los experimentales hubo un profesor especialista en ciencias y otro que no lo era; igualmente sucedió con su formación académica, uno era licenciado y el otro no.

Un quinto grupo $\left(\mathrm{C}_{0}\right)$ se añadió a la experimentación con el único propósito de constatar que los cambios producidos en los grupos eran debidos a las correspondientes intervenciones educativas y no al efecto de algún acontecimiento externo (descubrimientos científicos, polémicas aparecidas en los medios de comunicación, etc.) o a otras causas (inestabilidad de las actitudes, etc.).

Las características de cada grupo fueron:

$-\mathrm{C}_{0}$ : Grupo control que no recibió ninguna clase sobre genética. Del total de 24 adultos se pudieron recoger los datos completos de 16 de ellos. Éste fue el único criterio empleado para seleccionar la muestra en todos los grupos.

- $\mathrm{C}_{1}$ : Primer grupo control que sí recibió clases sobre genética. Del total de 12 adultos, $\mathrm{n}=10$. Profesorado: no especialista en ciencias.

$-\mathrm{C}_{2}$ : Segundo grupo control que recibió clases sobre genética. Del total de 21 adultos, $\mathrm{n}=13$. Profesorado: especialista en ciencias y con licenciatura en pedagogía.

$-E_{1}$ : Primer grupo experimental que aplicó el diseño de instrucción previsto. Del total de 35 adultos, $\mathrm{n}=19$. Profesorado: no especialista en ciencias.

$-\mathrm{E}_{2}$ : Segundo grupo experimental. Del total de 11 adultos, $\mathrm{n}=11$. Profesorado: investigador, especialista en ciencias y licenciado en psicología. 


\section{Técnicas estadísticas}

Para el tratamiento estadístico utilizamos las siguientes técnicas:

- Prueba $t$ de Student: Con ella averiguamos si, en cada clase, hubo una modificación significativa en las creencias, actitudes o intenciones tras el estudio del tema seleccionado. También se utilizó para comprobar la homogeneidad de las características de los sujetos de los distintos grupos.

- Análisis de varianza: Nos fue de utilidad para contrastar si hubo diferencias significativas en el cambio actitudinal logrado en los diferentes grupos.

- Análisis de covarianza: Realizado en cada grupo, nos permitió conocer si el cambio actitudinal es explicado por el aprendizaje logrado por cada diseño de instrucción o por otras variables diferentes a las experimentales.

\section{RESULTADOS}

Antes de presentar los resultados obtenidos de la comparación de medias, veamos los estadísticos descriptivos de cada grupo (Tabla I).

Iniciaremos el estudio estadístico comparando, mediante el análisis de varianza, las medias de las puntuaciones obtenidas por los sujetos en sus tres pruebas de actitudes (la puntuación global se obtuvo a partir de una composición aritmética de todos los ítems a excepción de los núm. 3, 4, 7 y 8, cuyas respuestas sólo podían interpretarse cualitativamente). Los resultados son los de la tabla II.

Por los resultados deducimos que no existen diferencias significativas entre sus actitudes iniciales ni entre las finales, pero sí las hay, y con gran significatividad (superior al 99\%), en el cambio actitudinal producido en los grupos experimentales.

Al partir de un modelo no equilibrado y de efectos fijos, comprobamos la homogeneidad de las varianzas, único aspecto, según Visauta y Batalle (1986), que puede tener importantes consecuencias en caso de incumplimiento. Y la prueba de Levene nos indica la falta de homocedasticidad en el caso de las actitudes netas. No obstante, las grandes diferencias existentes entre las desviaciones típicas de los grupos control y experimental y la alta significatividad nos llevan a pensar que, en este caso, el incumplimiento no debe tener ninguna repercusión importante. De hecho, al realizar la misma comparación mediante la técnica no paramétrica de Kruskal-Wallis, se mantuvo la significatividad de las diferencias (98\%).

También podría haberse comprobado que las actitudes finales de los sujetos experimentales son superiores a las actitudes de los grupos control (99\%) con sólo bloquear la cuantificación de las actitudes iniciales (Tabla III).
Tabla I

Datos descriptivos de las actitudes por grupo.

\begin{tabular}{|c|c|c|c|c|}
\hline \multicolumn{3}{|c|}{ NIVELES DE: CENTRO } & \multicolumn{2}{|c|}{ VARIABLE: AP } \\
\hline Variable & Valor & Media & Desv. Tipo & Casos \\
\hline \multicolumn{2}{|c|}{ Total de población } & 19,99 & 7,87 & 69 \\
\hline Centro & $\mathrm{C}_{0}$ & 22,25 & 1,39 & 16 \\
\hline Centro & $\mathrm{C}_{1}$ & 20,90 & 1,42 & 10 \\
\hline Centro & $\mathrm{C}_{2}$ & 21,54 & 1,08 & 13 \\
\hline Centro & $\mathrm{E}_{1}$ & 18,11 & 0,09 & 19 \\
\hline Centro & $\mathrm{E}_{2}$ & 17,27 & 9,90 & 11 \\
\hline \multicolumn{2}{|c|}{ NIVELES DE: CENTRO } & \multicolumn{3}{|c|}{ VARIABLE: AF } \\
\hline Variable & Valor & Media & Desv. Tipo & Casos \\
\hline \multicolumn{2}{|c|}{ Total de población } & 21,83 & 8,34 & 69 \\
\hline Centro & $\mathrm{C}_{0}$ & 21,56 & 6,56 & 16 \\
\hline Centro & $\mathrm{C}_{1}$ & 18,00 & 4,85 & 10 \\
\hline Centro & $\mathrm{C}_{2}$ & 21,08 & 6,20 & 13 \\
\hline Centro & $\mathrm{E}_{1}$ & 22,89 & 10,72 & 19 \\
\hline Centro & $\mathrm{E}_{2}$ & 24,73 & 10,37 & 11 \\
\hline \multicolumn{2}{|c|}{ NIVELES DE: CENTRO } & \multicolumn{3}{|c|}{ VARIABLE: CA } \\
\hline Variable & Valor & Media & Desv. Tipo & Casos \\
\hline \multicolumn{2}{|c|}{ Total de población } & 1,84 & 8,37 & 69 \\
\hline Centro & $\mathrm{C}_{0}$ & $-0,69$ & 6,45 & 16 \\
\hline Centro & $\mathrm{C}_{1}$ & $-2,90$ & 5,49 & 10 \\
\hline Centro & $\mathrm{C}_{2}$ & $-0,46$ & 5,52 & 13 \\
\hline Centro & $\mathrm{E}_{1}$ & 4,79 & 10,41 & 19 \\
\hline Centro & $\mathrm{E}_{2}$ & 7,45 & 8,04 & 11 \\
\hline
\end{tabular}

Antes de concluir que ese mayor incremento actitudinal es consecuencia del diseño instructivo, comprobamos que las diferencias no se deben a la heterogeneidad de los grupos en diversas variables (el bloqueo estadístico de las variables diferenciales, incluidas la ratio y el tiempo dedicado no varió los resultados según el acovar realizado). El efecto tampoco se debió totalmente al contenido instructivo, aunque sí fue un efecto significativo. Los acovar que se realizaron en cada grupo del cambio actitudinal logrado por el aprendizaje neto de conocimientos conceptuales y procedimentales (conocimientos finales menos conocimientos previos) y con las actitudes previas de covariante indicaron que, en los grupos control, el aprendizaje de conocimientos no tuvo ningún efecto significativo, mientras que en los grupos experimentales se alcanzaron significaciones superiores al 93 y $99 \%$. Esta tendencia se repitió al realizar ecuacio- 
Tabla II

Análisis de varianza de las pruebas de actitudes entre los cinco grupos $(\mathrm{N}=69)$.

\begin{tabular}{|c|c|c|c|c|c|c|}
\hline $\mathrm{FV}$ & & S C & $\mathrm{g}$ & M C & $\mathrm{F}$ & Homog. de Var: Levene \\
\hline AP & $\begin{array}{l}\text { Entre grupos } \\
\text { Intra grupos } \\
\text { Total }\end{array}$ & $\begin{array}{r}269,88 \\
3941,10 \\
4210,99\end{array}$ & $\begin{array}{r}4 \\
64 \\
68\end{array}$ & $\begin{array}{l}67,47 \\
61,58\end{array}$ & $1,10 \quad \mathrm{p}<.37$ & 0,69 sig. 0,60 \\
\hline $\mathrm{AF}$ & $\begin{array}{l}\text { Entre grupos } \\
\text { Intra grupos } \\
\text { Total }\end{array}$ & $\begin{array}{r}269,08 \\
4464,83 \\
4733,91\end{array}$ & $\begin{array}{r}4 \\
64 \\
68\end{array}$ & $\begin{array}{l}67,27 \\
67,76\end{array}$ & $0,96 \mathrm{p}<.43$ & 2,94 sig. 0,03 \\
\hline $\mathrm{CA}$ & $\begin{array}{l}\text { Entre grupos } \\
\text { Intra grupos } \\
\text { Total }\end{array}$ & $\begin{array}{r}907,79 \\
3857,45 \\
4765,25\end{array}$ & $\begin{array}{r}4 \\
64 \\
68\end{array}$ & $\begin{array}{r}226,95 \\
60,27\end{array}$ & $3,77 \mathrm{p}<.01$ & 2,96 sig. 0,03 \\
\hline
\end{tabular}

\begin{tabular}{|l|c|c|c|c|c|}
\hline \multicolumn{7}{|l|}{ ACOVAR AF por CENTRO } & SC & $\mathrm{g}$ & $\mathrm{MC}$ & $\mathrm{F}$ & Sig. F \\
\hline FV & 1156,99 & 1 & 1156,99 & 21,122 & 0,000 \\
\hline Variables covariantes & 1156,99 & 1 & 1156,99 & 21,122 & 0,000 \\
AP & & & & & \\
Efectos principales & 426,57 & 1 & 426,57 & 7,79 & 0,007 \\
CENTRO & 1347,72 & 2 & 673,86 & 12,30 & 0,000 \\
Explicado & 2738,81 & 50 & 57,78 & & \\
Residual & 4086,53 & 52 & 78,59 & & \\
Total & & & & \\
\hline
\end{tabular}

nes de regresión con el cambio actitudinal como variable dependiente y el aprendizaje neto de conocimientos como variable independiente, y se observó que, en los grupos control, el aprendizaje apenas alcanza a explicar un $1 \%$ (porcentaje no significativo), mientras que en los grupos experimentales logra explicar el $20 \%$ del cambio actitudinal. Se observa así que el efecto se mantiene debido al grupo (al diseño instruccional) y que el efecto del contenido es relativo, existiendo, por tanto, otras variables interventoras en dicho cambio en los grupos experimentales (Martínez Salvá, 1995).
A continuación, terminaremos de presentar los resultados obtenidos en cada grupo analizando puntualmente los cambios logrados en cada uno de ellos. Veamos previamente la tabla IV, en la que se reflejan las creencias y actitudes previas.

Las medias anteriores nos reflejan una situación inicial general en la que: a) la indiferencia hacia el tema de la ingeniería genética aplicada a personas (ítems 1 y 2) es bastante alta; $b$ ) se reconoce la responsabilidad de informarse y ser informado (ítems 4,5 y 6 ); $c$ ) no se posee 
Tabla IV

Medias y desviaciones típicas totales de cada variable del cuestionario previo de actitudes (AP) $\mathrm{N}=69$.

\begin{tabular}{|c|c|c|}
\hline ÍTEMS & MEDIA & DESV. T \\
\hline 1. Aceptar sin preocupaciones estos descubrimientos & 3,07 & 1,53 \\
\hline 2. Es complicado para interesarse por ellos & 3,39 & 1,43 \\
\hline 3. La genética genera inseguridad y peligro & 3,03 & 1,39 \\
\hline 4. Me preocupan posibles aberraciones genéticas & 3,87 & 1,41 \\
\hline 5. Tengo la responsabilidad de informarme sobre ello & 4,07 & 0,96 \\
\hline 6. Debe facilitarse información & 4,41 & 0,65 \\
\hline 7. Me falta información & 4,22 & 0,87 \\
\hline 8. Tengo una opinión clara & 2,52 & 1,09 \\
\hline 9. Se deben prohibir estas investigaciones & 2,72 & 1,35 \\
\hline 10. La genética puede mejorar la calidad de vida & 3,65 & 1,16 \\
\hline 11. Necesidad de limitar el uso para intereses particulares (de poder...) & 3,77 & 1,30 \\
\hline 12. Tengo derecho a opinar sobre este tema & 4,35 & 0,94 \\
\hline 13. Debo dar mi opinión; afecta a todos & 4,03 & 1,07 \\
\hline 14. Expresar opinión es poco útil & 3,48 & 1,47 \\
\hline 15. Mi opinión no tiene mucho valor & 3,06 & 1,29 \\
\hline 16. Puede que no dé mi opinión por nerviosismo & 3,25 & 1,49 \\
\hline 17. No tendría dificultad en convencer a otros de que opinen & 2,94 & 1,11 \\
\hline 18. La decisión (caso general) ha de contar con todos (los ciudadanos...) & 2,75 & 1,37 \\
\hline 19. Ídem (caso concreto) & 2,36 & 1,12 \\
\hline 20. Seguro que daría mi opinión & 3,38 & 1,40 \\
\hline TOTAL (AP) & 19,99 & 7,87 \\
\hline
\end{tabular}

información suficiente, ni opiniones formadas (ítems 7 y 8); d) no se manifiesta unanimidad hacia el uso solidario de la tecnología genética (ítems 9,10 y 11 ); $e$ ) existe una alta convicción sobre el derecho y la necesidad de expresar la opinión (ítems 12 y 13) en contraste con la baja confianza en la utilidad o valor de expresar la opinión (ítems 14 y 15) o en el convencimiento de no proporcionarla por causa de la ansiedad (ítem 16); f) poca predisposición a decidir solidariamente (ítems 18 y 19$)$.

Los resultados siguientes vienen a reflejar en qué items se ha producido un cambio significativo en cada grupo tras la intervención.

\section{CONCLUSIONES}

De estos resultados se desprenden las siguientes observaciones:
1. La ausencia de cambios en el grupo de control $\mathrm{C}_{0}$ (que no recibió ninguna información sobre el contenido experimental) indica la estabilidad de las mediciones y que no hubo acontecimientos externos (a nivel de medios de comunicación, por ejemplo) que pudieran haber influido en las actitudes manifestadas.

2. Tratar el tema en clase fue suficiente para la modificación de las manifestaciones actitudinales hacia la participación ciudadana en la toma de decisiones, aunque sólo en ciertos aspectos puntuales.

3. Tales modificaciones pueden ir incluso en sentido contrario a la participación ciudadana cuando no se trabaja ésta de un modo explícito, algo que sucedió especialmente en el grupo $\mathrm{C}_{1}$ cuando, por ejemplo, descendió significativamente la creencia en la obligación a participar en la toma de decisiones (también estuvo cerca de conseguirse un cambio negativo significativo de su actitud global, su significatividad fue del $87 \%)$, mientras que, en el otro grupo control $\left(\mathrm{C}_{2}\right)$, los 
Tabla V

Medias y prueba $T$ de Student $(P / F)$ por grupos. Se presentan sólo variables con cambio significativo (90\%; * al 95\%)

\begin{tabular}{|c|c|c|c|c|}
\hline & ÍTEM & $\begin{array}{c}\text { MEDIAS } \\
\text { A / D }\end{array}$ & $\mathrm{t}$ & Sig. \\
\hline $\mathrm{C}_{0}$ & - & & & \\
\hline $\mathrm{C}_{1}$ & $\begin{array}{l}\text { 2. El tema es complicado para interesarse } \\
\text { 4. Me preocupan posibles aberraciones } \\
\text { 5. Tengo la responsabilidad de informarme } \\
\text { 6. Debe facilitarse información } \\
\text { 7. Me falta información } \\
\text { 12. Tengo derecho a opinar } \\
\text { 13. Debo dar mi opinión, afecta a todos } \\
\text { 17. Sin dificultades para convencer a otros }\end{array}$ & $\begin{array}{l}4,00 / 3,50 \\
4,60 / 3,70 \\
4,50 / 4,00 \\
4,60 / 4,00 \\
4,60 / 2,80 \\
4,60 / 4,10 \\
4,60 / 3,80 \\
3,20 / 2,20\end{array}$ & $\begin{array}{l}2,24 \\
2,21 \\
3,00 \\
1,96 \\
4,07 \\
1,86 \\
3,21 \\
2,74\end{array}$ & $\begin{array}{l}, 052 \\
, 054 \\
, 015 * \\
, 081 \\
, 003 * \\
, 096 \\
, 011 * \\
, 023 *\end{array}$ \\
\hline $\mathrm{C}_{2}$ & $\begin{array}{l}\text { 1. Son descubrimientos a aceptar sin preocuparse } \\
\text { 12. Tengo derecho a opinar }\end{array}$ & $\begin{array}{l}3,00 / 2,15 \\
4,38 / 3,62\end{array}$ & $\begin{array}{l}1,88 \\
2,13\end{array}$ & $\begin{array}{l}, 085 \\
, 054\end{array}$ \\
\hline $\mathrm{E}_{1}$ & $\begin{array}{l}\text { 1. Son descubrimientos a aceptar sin preocuparse } \\
\text { 4. Me preocupan posibles aberraciones } \\
\text { 7. Me falta información } \\
\text { 8. Tengo una opinión clara } \\
\text { 13. Debo dar mi opinión, afecta a todos } \\
\text { 17. Sin dificultades para convencer a otros } \\
\text { 19. Cómo tomar decisiones (caso concreto) } \\
\text { 20. Estoy seguro que daría mi opinión } \\
\text { Total }\end{array}$ & $\begin{array}{r}3,16 / 2,47 \\
3,05 / 4,47 \\
4,32 / 3,68 \\
2,47 / 3,05 \\
3,42 / 4,63 \\
2,48 / 3,63 \\
2,11 / 2,89 \\
3,05 / 3,89 \\
18,1 / 22,89\end{array}$ & $\begin{array}{r}2,00 \\
-3,62 \\
2,05 \\
-1,77 \\
-4,15 \\
-2,80 \\
-2,04 \\
-2,11 \\
-2,01\end{array}$ & $\begin{array}{l}, 061 \\
, 002 * \\
055 \\
, 094 \\
, 001 * \\
, 012 * \\
, 056 \\
, 049 * \\
, 060\end{array}$ \\
\hline $\mathrm{E}_{2}$ & $\begin{array}{l}\text { 3. Sensación de inseguridad por los avances en genética } \\
\text { 14. Mi opinión es poco útil } \\
\text { 16. Puede que no dé mi opinión por nerviosismo } \\
\text { 19. Cómo tomar decisiones (caso concreto) } \\
\text { 20. Estoy seguro que daría mi opinión } \\
\text { TOTAL }\end{array}$ & $\begin{array}{l}2,55 / 3,82 \\
4,00 / 2,45 \\
3,45 / 4,18 \\
2,27 / 3,18 \\
3,09 / 4,09 \\
17,3 / 24,7\end{array}$ & $\begin{array}{r}-2,22 \\
3,02 \\
-2,39 \\
-2,65 \\
-1,98 \\
-3,07\end{array}$ & $\begin{array}{l}, 051 \\
, 013 * \\
, 038 * \\
, 024 * \\
, 076 \\
, 012 *\end{array}$ \\
\hline
\end{tabular}

resultados son más escasos y contradictorios. Es importante destacar que en ambos grupos descendió la convicción del derecho a opinar (ítem 12).

4. Los cambios logrados con el enfoque comunicativo (grupos $\mathrm{E}_{1}$ y $\mathrm{E}_{2}$ ) no fueron significativos en todos los aspectos considerados, aunque fueron siempre en el sentido de la participación solidaria y responsable (a excepción del ítem 16, que en el grupo $\mathrm{E}_{2}$ indicó una mayor resistencia a participar a causa de la ansiedad). También se consiguió en los dos grupos un incremento significativo de la actitud tomada globalmente. Destaca, por otra parte, el incremento de sentimientos de inseguridad, ansiedad y preocupación, al contrario de lo que sucede en el grupo $\mathrm{C}_{1}$.

5. En ninguno de los grupos se consiguió cambiar a favor de los usos solidarios de las aplicaciones de la ingeniería genética (ítems 9 y 10), ni en el valor de la opinión propia (ítem 15), ni en la necesidad de contar con la opinión de los ciudadanos cuando se plantea una decisión (ítem 18, caso general).

A partir de estos resultados concluimos que es necesario un enfoque comunicativo de los contenidos de ciencias para evitar el riesgo de generar actitudes contrarias a la participación ciudadana. También, contemplando que no se consiguieron cambios generalizados en todas las creencias y actitudes consideradas, comprendemos que no es suficiente con un tratamiento puntual sino que la reflexión y generalización de las actitudes participativas requiere un tratamiento educativo más amplio. Pensamos que muchos de los contenidos del currículo de la FPA admiten trabajar la dimensión participativa y que la misma convivencia en el centro (órganos de gobierno, actividades de centro, etc.) es una gran oportunidad para estimular este derecho y esta responsabilidad.

Por otra parte, los resultados también nos advierten de una posible dificultad: el aumento del estrés. Sin duda el miedo, la falta de confianza en uno mismo, la escasez de recursos o habilidades sociales y algunos otros factores pueden obstaculizar la conducta solidaria y responsable. Por todo ello, consideramos que un diseño instructivo o, mejor, toda la acción educativa necesita reforzar a la persona en aspectos como las técnicas y recursos de afrontamiento del estrés, autoestima, asertividad y otras habilidades sociales que son también importantes para que la alfabetización científica consiga manifestarse plenamente. 


\section{REFERENCIAS BIBLIOGRÁFICAS}

APEL K.O. (1988). En Cortina, A. Razón comunicativa y responsabilidad solidaria. Salamanca: Sígueme.

BORREGUERO, P. (1993). STS: una aproximación empírica a las relaciones ciencia-tecnología-sociedad. Tesis de licenciatura. Facultad de Psicología de Valencia.

CORTINA, A. (1994). Ética aplicada y democracia radical. Madrid: Tecnos.

ESCÁMEZ, J. (1986). La enseñanza de las actitudes y valores. Valencia: Nau Llibres.

FAURE, E. et al. (1981). Aprender a ser. Madrid: Alianza Universidad.

HABERMAS, J. (1987) Teoría de la acción comunicativa I. Racionalidad de acción y racionalización social. Madrid: Taurus.

MARTÍNEZ GUZMÁN, V. (1993). Explicitación de la racionalidad europea, en Teoria de Europa. Valencia: Nau Llibres.

MARTÍNEZ SALVÁ, F.A. (1995). Alfabetización científica en la formación de personas adultas a partir del MISE: un análisis experimental. Tesis doctoral. Facultad de Psicología de la Universidad de Valencia.

MASON, R. (1989). Adults and Science. Adults Learning, Vol. 1(2), pp. 37-39.

MORA, C. (1991). Switching on to Science. Adult Learning, Vol. 3(4), pp. 96-98.

SANJOSÉ, V., SOLAZ, J.J. y VIDAL-ABARCA, E. (1993). Mejorando la efectividad instruccional del texto educativo en ciencias: primeros resultados. Enseñanza de las Ciencias, 11(2), pp. 137-148.

SCANDRETT, E. (1995). Learning Science in Context. Adults Learning, Vol. 5(10), pp. 258-263.

STRACHAN, R., BROWN, J. y SCHULLER, T. (1988). Adult education and scientific literacy: an innovation with workshops. Adult Education, Vol. 61(2), pp. 109-114.

VISAUTA, B. y BATALLE, P. (1986). Métodos estadísticos aplicados. Tomo II. Barcelona: PPU.

[Artículo recibido en mayo de 1996 y aceptado en diciembre de 1997.] 
ANEXO 1

Grupo: Centro:

En la presente investigación pretendemos conocer qué opinión te merecen los avances científicos en GENÉTICA HUMANA. Estas contestaciones serán confidenciales y reservadas, además no afectarán para nada tus notas.

Si decides colaborar con este estudio, contestarás las preguntas siguientes redondeando con un círculo la respuesta más adecuada. Si deseas anularla, táchala con una $X$ y vuelve a contestar.

\section{GRACIAS POR TU COLABORACIÓN}

\section{INGENIERÍA GENÉTICA APLICADA A PERSONAS}

Gracias a la investigación del genoma humano se podrá conocer el mapa genético de la especie humana. Esto permitiría avanzar en diversas aplicaciones: detectar y evitar enfermedades hereditarias, modificar las características de una persona (su aspecto u otras cualidades), etc.

\section{RESPECTO A ESTE TEMA:}

1. Pienso que son descubrimientos como otros muchos que se producen todos los días y que hay que aceptar sin muchas preocupaciones.
a) Muy de acuerdo
b) Bastante
c) Poco
d) Nada de acuerdo
e) No lo sé

2. Tengo la impresión de que este tema es algo complicado para que una persona normal (no científica) pueda entenderlo e interesarse por él.

3. Me siento inseguro y en peligro ante estos avances que no sé cómo pueden afectarnos en un futuro.

4. Me preocupa mucho que puedan producir aberraciones genéticas con las personas (darles un aspecto artificial, como robots, monstruos...).

5. Tengo la responsabilidad de informarme sobre estas investigaciones.

6. Los científicos, los medios de comunicación (televisión, prensa, etc.) y los centros educativos y culturales deberían favorecer la comprensión de estos temas.

7. Me falta información para tener una opinión sobre este tema.

8. Tengo suficientes ideas claras sobre lo que se debe y no se debe hacer, en general, en ingeniería genética humana.

9. Debemos dejar que la naturaleza siga su curso y prohibir las investigaciones sobre genética humana.

10. Creo que la ingeniería genética puede mejorar nuestra salud y, por tanto, nuestra calidad de vida.

11. Creo que es necesario limitar legalmente el desarrollo de estas investigaciones para evitar que se pueda manipular a las personas para intereses particulares (económicos, de poder...).

12. Como ciudadano tengo derecho a opinar sobre estas aplicaciones de la genética en personas.

13. Debo dar mi opinión sobre la ingeniería genética aplicada a las personas porque estoy convencido de que nos afecta e interesa a todos.

14. Dar mi opinión es poco útil porque deciden los científicos o las personas que tienen el poder político o económico.

15. Creo que, sobre este tema, mi opinión no tiene mucho valor.

16. Es posible que, sobre este tema, no diera mi opinión en público porque no estoy acostumbrado/a y me pongo muy nervioso/a.

17. Sobre este tema, creo que no tendría dificultades en convencer a las personas más importantes y próximas a mí de la conveniencia de dar públicamente nuestra opinión.

18. ¿Quiénes, crees tú, que deberían decidir sobre la ingeniería genética aplicada a personas?, ¿los científicos, los políticos, los jueces, los filósofos, los empresarios que invierten el dinero en la investigación, los ciudadanos...? 
19. Si se descubriera cómo proporcionar a los embriones humanos más fuerza física e inteligencia, ¿qué crees que debería hacer el Gobierno?

a) No hacer caso.

b) Aplicarlo inmediatamente.

c) Decidir su aplicación conjuntamente con los expertos (científicos, psicólogos, filósofos, jueces, etc.), ya que son los que poseen más información.

d) Decidir su aplicación, conjuntamente, los expertos y todos los grupos políticos porque son los responsables de representar nuestros intereses.

e) Decidir su aplicación, conjuntamente, expertos, grupos políticos y la ciudadanía.

f) No lo sé.

20. En el caso anterior, estoy seguro de que daría mi opinión de alguna manera: asistiendo a reuniones, dando mi conformidad si estuviera de acuerdo, acudiendo a alguna manifestación, etc. 